\title{
Análisis factorial confirmatorio de Indicadores de Progreso de Aprendizaje en Matemáticas (IPAM) en escolares de primer curso de Primaria
}

\author{
Juan E. Jiménez y Sara del Cristo de León \\ Universidad de La Laguna (España)
}

\begin{abstract}
En este estudio se pretende poner a prueba un modelo hipotético para profundizar en la dimensionalidad del sentido numérico utilizando la técnica del Análisis Factorial Confirmatorio (AFC). Se ha llevado a cabo un estudio longitudinal con una muestra de 176 alumnos de primer curso de Educación Primaria de las Islas Canarias, a los que se les administró el instrumento Indicadores de Progreso de Aprendizaje en Matemáticas (IPAM) en tres momentos diferentes del curso escolar. El IPAM es un instrumento CBM compuesto por tres medidas alternativas o paralelas (A, B y C) que tratan de medir una misma estructura latente (i.e., sentido numérico). Estas medidas se aplican en tres momentos diferentes a lo largo del año escolar (i.e., noviembre, febrero, mayo). Su principal objetivo es el cribado universal y la evaluación del progreso de aprendizaje en matemáticas del alumnado de educación primaria mediante la evaluación de la fluidez en la resolución de diferentes tareas (i.e., comparación de magnitudes, operaciones de un dígito, operaciones de dos dígitos, series numéricas, y valor de posición). La fluidez es medida contabilizando el número de respuestas correctas que da el alumno en un tiempo dado. Los resultados del AFC confirman un buen ajuste del modelo propuesto para los distintos momentos de medida.
\end{abstract}

Palabras clave: Sentido numérico, educación primaria, matemáticas, análisis factorial confirmatorio, medida basada en el currículo.

Confirmatory factor analysis of Mathematics Progress Indicators (IPAM) in first-grade students. This study analyses the factorial structure of Mathematics Progress Indicators (IPAM), by using Confirmatory Factorial Analysis (CFA) techniques. For this purpose, a longitudinal study was carried out with a sample of 176 first-grade students from the Canary Islands, IPAM was administered to the study sample three times throughout the scholar year. The IPAM is a Curriculum-Based Measurement (CBM) instrument composed of three alternative or parallel measures (A, B and C) that try to measure the same latent structure (i.e., number sense). The IPAM measures were administered three times per year (i.e., fall, winter, spring). Its main objective is the universal screening and evaluation of students' mathematics learning progress in the elementary grades through the evaluation of fluency for different tasks (i.e., magnitude comparison, one-digit operations, two-digit operations, missing number, and position value). Fluency is measured by counting the number of correct answers given by the student at a given time. The results of the CFA confirm a good fit of the proposed model for the different moments of measurement.

Keywords: Number sense, elementary grade, mathematics, confirmatory factor analysis, curriculum-based measurement.

Correspondencia: Juan E. Jiménez. Facultad de Psicología. Universidad de La Laguna. Campus de Guajara, S/N. C.P.: 38200. La Laguna, Islas Canarias (España).E-mail: ejimenez@ull.es 
El sentido numérico se convirtió en foco de atención en los años 90, y desde entonces la evidencia empírica recogida a través de múltiples investigaciones muestra que es una habilidad clave para el aprendizaje de las matemáticas (Aubrey, Dahl, y Godfrey, 2006; Aunio y Niemivirta, 2010; Baker, Gersten, y Lee, 2002; Geary, 2013; Jordan, Kaplan, Locuniak, y Ramineni, 2007; Kolkman, Kroesbergen, y Leseman, 2013; LeFevre, Fast, Skwarchuk, Smith-Chant, Bisanz, Kamawar, et al., 2010; Sasanguie, Göbel, Moll, Smets, y Reynvoet; 2013; Sood y Jitendra, 2007). Diversos autores han demostrado que el sentido numérico es una habilidad que se desarrolla de forma muy temprana (Barth, Beckmann, y Spelke, 2008; Xu y Arriaga, 2007), permitiendo a los niños comparar conjuntos de números pequeños entre sí, discriminando entre el mayor y el menor (Bruer, 1997; Nieder y Dehaene, 2009). A esta habilidad para ser capaces de reconocer de manera automática e instantánea el tamaño de pequeños conjuntos de objetos sin contar, es a lo que se conoce como subitización (del inglés subitising), la cual ha sido observada en niños pequeños como una habilidad básica para la futura comprensión del número (Conderman, Jung, y Hartman, 2014; Jung, Hartman, Smith, y Wallace, 2013). En este sentido, Gersten y Chard (1999) propusieron que el sentido numérico temprano podría ser a la matemática, lo que la conciencia fonémica es a la lectura. Ellos establecieron un paralelismo con la lectura en el sentido de que del mismo modo que los déficits en las habilidades tempranas de conciencia fonémica pueden producir un posterior fracaso en lectura; los déficits tempranos en las habilidades relacionadas con la adquisición del sentido numérico, podrían predecir posteriores dificultades de aprendizaje en matemáticas (DAM).

De hecho, el rol del sentido numérico en las DAM también ha sido apoyado por múltiples investigaciones donde se ha demostrado que las DAM resultan primariamente de déficits en procesamiento numérico (Mazzocco y Thompson, 2005). Asimismo, Gersten, Jordan, y Flojo (2005) demostraron que la presencia de dificultades a la hora de recuperar de forma rápida hechos numéricos estaba vinculada con el desarrollo del sentido numérico, siendo un rasgo consistente en los estudiantes con DAM.

No obstante, definir el sentido numérico ha supuesto diversos debates en la comunidad científica. El National Council of Teachers of Mathematics (NCTM, 2000) estableció que éste hace referencia a la habilidad para entender el significado de los números, las relaciones existentes entre ellos, reconocer el tamaño relativo de los números, su uso para medir objetos y eventos, y pensar de forma flexible. De forma más específica, el National Mathematics Advisory Panel (NMAP) (2008) define el sentido numérico como un constructo en evolución, discriminando entre el sentido numérico adquirido de forma informal y el que se adquiere de manera formal. El primero de ellos hace referencia a la habilidad para identificar de manera inmediata el valor numérico asociado a pequeñas cantidades, desarrollar habilidades básicas de conteo, calcular de manera aproximada la magnitud de pequeños conjuntos de objetos y resolver operaciones numéricas sencillas. Una concepción más avanzada del sentido numérico sería aquella que los niños adquieren 
a través de la instrucción formal y requiere la comprensión del valor de posición, de como todos los números pueden ser compuestos y descompuestos, y del significado de las operaciones aritméticas básicas de adición, substracción, multiplicación y división. Esto también requiere la comprensión de las propiedades conmutativa, asociativa y distributiva y el conocimiento de cómo aplicar esos principios a la resolución de problemas.

Son diversos los autores que apoyan esta subdivisión del sentido numérico, haciendo referencia a un sentido numérico más temprano, que los niños normalmente adquieren de manera informal (Ivrendi, 2011; Jordan y Levine, 2009) antes de iniciarse en el sistema educativo, siendo éste un importante prerrequisito para el posterior logro matemático (Andrews y Sayers, 2015; Gersten y Chard, 1999).

Tomando en consideración las distintas definiciones que se han realizado del sentido numérico, se hace evidente que este constructo es bastante amplio, estando compuesto por múltiples habilidades. Así, atendiendo a lo expuesto, estas habilidades se relacionarían con: (1) magnitudes numéricas, (2) operaciones numéricas, (3) valor de posición, (4) conteo, y (5) línea numérica.

\section{¿Cómo medir el sentido numérico?}

Un cuerpo amplio de bibliografía científica aporta evidencia de que las medidas basadas en el sentido numérico tienen el potencial de proveer de indicadores técnicamente adecuados para la evaluación del logro académico en matemáticas de los estudiantes (Andrews y Sayers, 2015; Foegen, Jiban, y Deno, 2007; Lembke y Foegen, 2009). De forma más específica, se ha encontrado que las medidas basadas en el currículo (del inglés, Curriculum Based Measurement, CBM) han demostrado adecuación técnica para detectar la mejora del logro académico del alumno (Fuchs y Fuchs, 1986; Stecker, Fuchs, y Fuchs, 2005) y predecir el estatus de riesgo (Lembke y Foegen, 2009). Estas herramientas han sido empleadas como medidas de cribado universal (Kelley, Hosp, y Howell, 2008) para la detección de alumnado en riesgo de presentar dificultades de aprendizaje en el área de las matemáticas.

Son varios los autores que han diseñado herramientas de cribado basadas en el sentido numérico, encontrando que las tareas que incluyen la discriminación de cantidades, identificación numérica, secuencias y cálculo son buenos predictores del estatus de riesgo (Foegen et al., 2007; Lembke y Foegen 2009). Teniendo en cuenta lo expuesto hasta ahora se ha diseñado la medida Indicadores de Progreso de Aprendizaje en Matemáticas (IPAM) (Jiménez y de León, 2016), la cual incluye tareas de comparación de magnitudes, secuencias numéricas, operaciones de uno y dos dígitos, y valor de posición.

El objetivo de este trabajo es poner a prueba un modelo hipotético para profundizar en la dimensionalidad del sentido numérico mediante la técnica de Análisis Factorial Confirmatorio (AFC). Esto nos permitirá conocer en qué medida el constructo 
sentido numérico explica las correlaciones entre las tareas que componen la herramienta IPAM (i.e., comparación de magnitudes, operaciones de un dígito, operaciones de dos dígitos, series numéricas, y valor de posición).

\section{MÉTODO}

\section{Participantes}

La población de este estudio corresponde a alumnado de $1^{\circ}$ de Educación Primaria perteneciente a cinco centros de Educación Primaria, uno concertado y cuatro públicos, de la provincia de Santa Cruz de Tenerife. La muestra incluyó un total de 176 alumnos, de los cuales 84 eran niños y 92 eran niñas. La edad promedio de la muestra fue de 6.2, con un rango de edad de 5.5 a 6.8 años. Se excluyó de la muestra al alumnado con necesidades educativas especiales (i.e., déficit sensorial, intelectual, físico o motor).

\section{Instrumentos}

Se utilizó la herramienta Indicadores de Progreso de Aprendizaje en Matemáticas (IPAM) (Jiménez y de León, 2016) para primero de Educación Primaria. El IPAM es un instrumento CBM compuesto por 3 medidas alternativas o paralelas (A, B y C) que tratan de medir una misma estructura latente (i.e., sentido numérico). Estas medidas se aplican en tres momentos diferentes a lo largo del año escolar, siendo los meses correspondientes de aplicación Noviembre (Forma A), Febrero (Forma B), y Mayo (Forma C). Su principal objetivo es el cribado universal y la evaluación del progreso de aprendizaje del alumnado mediante la evaluación de la fluidez en la resolución de cada tarea. La fluidez es medida contabilizando el número de respuestas correctas que da el alumno en un tiempo dado.

Esta prueba está compuesta por cinco tareas, que se aplican de forma consecutiva con una duración máxima de dos minutos por tarea. En la primera tarea, comparación de magnitudes $(\mathrm{CN})$, al alumnado se le presentan una serie de parejas de números, debiendo identificar aquel que represente mayor magnitud en cada una de las parejas dadas. La segunda tarea, operaciones de dos dígitos (ODD), requiere que el alumno resuelva de forma correcta operaciones de adición y sustracción que combinan números de un dígito y de dos dígitos. En la tercera tarea, secuencias numéricas (SN), al alumno se le presentan una serie de secuencias compuestas por dos dígitos y una incógnita, la cual el alumno deberá completar con el número correspondiente. La cuarta tarea, operaciones de un dígito (OUD), se basa en la resolución de operaciones básicas de adición y sustracción, en las que se emplean números de 1 al 9. Finalmente, en la quinta tarea, valor de posición (VP), a partir de una representación gráfica dada el alumno deberá deducir el número representado, escribiéndolo a continuación. 


\section{Procedimiento}

Previo a la administración del instrumento, se solicitó autorización al centro y a las familias para la realización del estudio. Para llevar a cabo la recogida de información se entrenó a 7 licenciados en Psicología y 2 licenciados en Psicopedagogía en la aplicación de la herramienta IPAM. Las tareas del IPAM se contrabalancearon, de tal manera que en cada uno de los centros participantes se presentaron las tareas en un orden distinto, teniendo la aplicación de la prueba una duración media de 15 minutos. Las pruebas se aplicaron en tres momentos diferentes a lo largo del año escolar (noviembre, febrero, mayo), con un intervalo aproximado de tres meses entre cada una de ellas. La aplicación se llevó a cabo de forma colectiva en el grupo clase con un promedio de 20 alumnos por aula y en horario lectivo, informando al alumnado del objetivo del estudio y su confidencialidad.

\section{Análisis de datos}

En este estudio se postula un modelo hipotético del sentido numérico mediante un AFC basado en los datos empíricos obtenidos a través de las respuestas proporcionadas por el alumnado en la herramienta IPAM. El modelo examinado es aquel en el que se postula la existencia de un único factor de primer orden, y que por tanto todas las tareas que incluye la herramienta están relacionadas con ese factor. Este modelo corresponde a una concepción teórica unidimensional del sentido numérico.

En una primera fase hemos formulado la estructura de relaciones entre los indicadores observables y el factor latente. Un segundo paso ha consistido en emplear la metodología de modelación de ecuaciones estructurales SEM, implementada en el paquete LISREL 8.8, en la modalidad de Análisis Factorial Confirmatorio [AFC] (Jöreskog y Sörbom, 1996-2001). Este procedimiento consiste en analizar la estructura de covarianza en la base de datos de los 5 indicadores observables y tratar de extraer evidencia de validez para afirmar que el modelo de medición coincide con la estructura conceptual postulada en la teoría.

La fase de especificación del modelo incluye una representación gráfica de la estructura teórico-conceptual del instrumento bajo análisis. Al tratarse el IPAM de una herramienta formada por tres medidas, una representación gráfica de cada una de ellas es presentada en el análisis de los resultados en las figuras 1-3.

En cada una de estas figuras, el círculo representa el factor latente (constructo) y los óvalos los cinco indicadores observables (tareas). Las flechas unidireccionales representan las líneas de influencia del factor sobre los respectivos indicadores observables. Estas especificaciones de direccionalidad equivalen a afirmar que cada uno de los indicadores relacionados mide la dimensión a la que en teoría pertenecen. Por tanto, la parte que estas cinco tareas comparten entre sí puede atribuirse a la existencia de una estructura latente - en este caso, configurada en torno a un solo factor- que explica 
(varianza explicada) las correlaciones entre las tareas. Finalmente, las flechas unidireccionales que aparecen a la derecha de los indicadores observables, representan los términos de error que son la influencia de fuentes de variabilidad única (o idiosincrática) asociadas a cada indicador observable.

Se utilizó el Software R para el cálculo de estadísticos descriptivos y los coeficientes de correlación de los resultados obtenidos en las tareas, los cuales se calcularon con el fin de evaluar la ausencia de cualquier efecto de multicolinealidad entre los indicadores a estudiar. Se calculó la media, desviación típica, máximo, mínimo, asimetría y curtosis para caracterizar los datos de la muestra.

Mediante el software R y ULLRToolbox, se evaluó la validez factorial de este modelo a través de un AFC. La fiabilidad compuesta (CR) y la varianza media extraída (AVE) para cada factor se calculó como describen Fornell y Larcker (1981). La existencia de los valores atípicos se evaluó por la distancia al cuadrado de Mahalanobis (D2) y la normalidad de los indicadores se evaluó mediante los coeficientes de asimetría (SK) y curtosis $(\mathrm{Ku})$ uni y multivariante. Se utilizó el método de estimación de máxima verosimilitud (ML). La evaluación del método se realizó mediante los siguientes índices: chi cuadrado $(\chi 2)$, cuadrados relación chi estadísticos / grados de la libertad $(\chi 2 / d f)$, índice de ajuste comparativo (CFI), la bondad de ajuste del modelo (GFI), y el error cuadrático medio de aproximación (RMSEA), P [RMSEA $\leq 0,05]$.

\section{RESULTADOS}

Debido a que la herramienta IPAM está compuesta por tres formas, se expondrán a continuación los resultados para cada una de estas formas.

\section{IPAM Forma A}

En la Tabla 1 presentamos los análisis estadísticos descriptivos de cada una de las tareas que componen el IPAM (Forma A). Se muestran los valores máximo y mínimo, medias, desviación estándar, asimetría y curtosis para cada tarea. Un primer análisis de estos datos muestra que los valores de asimetría y curtosis son cercanos a cero. Por tanto, se evidencia una distribución normal y una dispersión satisfactoria de los resultados, con la excepción de ODD y VP, las cuales tienden a valores más altos en relación al promedio, aunque se mantienen dentro de los rangos aceptables para estos estadísticos. Si observamos los valores de simetría y curtosis de todas las variables del estudio podemos observar que no existen problemas con la distribución de los datos puesto que los valores absolutos de estos índices no exceden los valores 3.0 y 10.0 respectivamente (Kline, 2005). Las desviaciones en las puntuaciones estándar se sitúan entre 9.75 y 2.42. 
La matriz de correlación de los resultados obtenidos en las diferentes tareas que componen el IPAM (Forma A) se presenta en la tabla 2. Los resultados obtenidos muestran correlaciones estadísticamente significativas entre todos los indicadores.

Tabla 1. Estadísticos descriptivos de IPAM Forma A

\begin{tabular}{lcccccc}
\hline & Mínimo & Máximo & Media & Desviación estándar & Asimetría & Curtosis \\
\hline CN & 1 & 50 & 23.88 & 9.75 & -.03 & -.12 \\
\hline ODD & 0 & 11 & 2.41 & 2.42 & 1.31 & 1.57 \\
\hline SN & 0 & 20 & 8.34 & 4.31 & -.02 & -.10 \\
\hline OUD & 0 & 20 & 7.32 & 3.65 & .24 & -.15 \\
\hline VP & 0 & 18 & 2.75 & 2.74 & 2.15 & 5.97 \\
\hline Nota. CN=Comparación de magnitudes, ODD=Operaciones de dos dígitos, SN=Series numéricas, \\
$\begin{array}{l}\text { OUD=Operaciones de un dígito, VP=Valor de posición. IPAM=Indicadores de Progreso de } \\
\text { Aprendizaje en Matemáticas. }\end{array}$
\end{tabular}

Tabla 2. Coeficientes de correlación entre las tareas de IPAM Forma A

\begin{tabular}{llllll}
\hline & CN & ODD & SN & OUD & VP \\
\hline CN & 1.00 & $.49^{* *}$ & $.61^{* *}$ & $.60^{* *}$ & $.38^{* *}$ \\
\hline ODD & & 1.00 & $.54^{* *}$ & $.58^{* *}$ & $.48^{* *}$ \\
\hline SN & & & 1.00 & $.59^{* *}$ & $.38^{* *}$ \\
\hline OUD & & & & 1.00 & $.53^{* *}$ \\
\hline VP & & & & & 1.00 \\
\hline
\end{tabular}

Nota. $\mathrm{CN}=$ Comparación de magnitudes, ODD=Operaciones de dos dígitos, $\mathrm{SN}=$ Series numéricas, $\mathrm{OUD}=$ Operaciones de un dígito, $\mathrm{VP}=$ Valor de posición. IPAM=Indicadores de Progreso de Aprendizaje en Matemáticas.

$* * p<.001$

En primer lugar, el AFC produce una representación de las relaciones estructurales observadas en la matriz derivada. Los valores que representan las flechas unidireccionales son las líneas de influencia entre el factor latente y los indicadores observables y se interpretan como coeficientes de regresión multivariada. Estos han sido calculados por el programa al analizar la matriz de covarianza derivada de los indicadores observables. Se calculan los coeficientes de regresión entre el factor latente y los indicadores observables. El primer valor que se encuentra es 0.61 , el cual corresponde a la influencia del factor latente Sentido Numérico sobre el indicador observable CN. El coeficiente de regresión presenta signo positivo, lo que evidencia una relación directa entre el factor latente y el indicador observable. Esto implica que cuando el factor latente aumenta una unidad, el indicador $\mathrm{CN}$ aumenta una proporción de 0.61. La misma interpretación debe realizarse con las relaciones existentes entre el factor latente y el resto de indicadores observables, encontrándose un coeficiente de 0.76 para ODD, un coeficiente de 0.68 para SN, un coeficiente de 0.80 para OUD, y un coeficiente de 0.64 para VP (ver figura 1).

Estos resultados muestran que la evaluación preliminar del modelo verifica que los coeficientes derivados presentan el sentido positivo esperado de acuerdo con la teoría empleada en la configuración del modelo de medida. Posteriormente se analizó el nivel de 
ajuste del modelo propuesto con el modelo estudiado. En primer lugar, se obtiene el valor de $\chi^{2}$, el cual resultó igual a 2.255 ( $\left.p=0.689\right)$, implicando que el modelo y los resultados se ajustan entre sí. Este resultado necesita ser confirmado, para ello se procede a la evaluación del ajuste del modelo mediante los índices de bondad de ajuste. Cuando se examina el grado de ajuste que ofrece este modelo a los datos empíricos, los resultados indican que el modelo presenta un buen ajuste a los datos $\left(\mathrm{SRMR}=0.019 ; \chi^{2}=2.255 ; d f=4\right.$; pvalue=0.689; NFI=0.99; $\mathrm{CFI}=1 ; \mathrm{GFI}=0.993 ; \mathrm{AGFI}=0.974 ; \mathrm{RMSEA}=0 ; \mathrm{IC}=0.00-0.10)$.

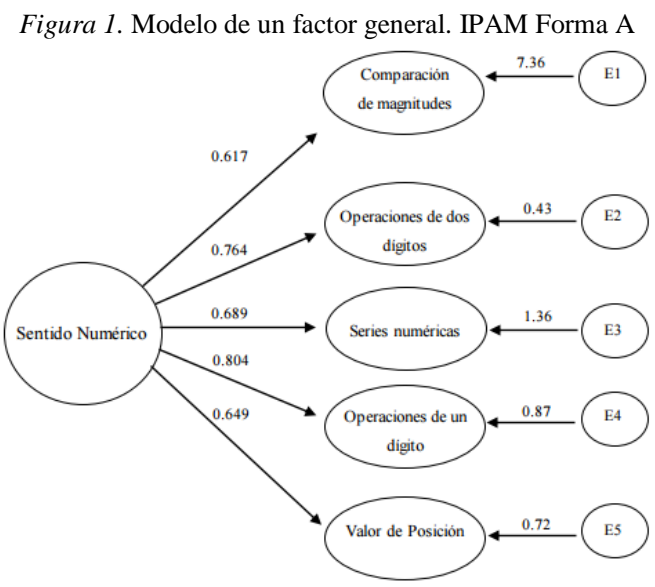

Para conseguir un ajuste adecuado del modelo fue necesario liberar la covarianza de error entre los indicadores observables CN y SN. Se estima que esta covarianza de error puede estar explicada por el uso, por parte del alumnado, de un procedimiento de trascodificación. Desde la perspectiva del Modelo de Triple Código (Dehaene y Cohen, 1995; Dehaene, Piazza, Pinel, y Cohen, 2003), la información numérica se puede manipular a través de tres tipos de códigos: código análogo a las magnitudes o analógico, código verbal-auditivo, y código arábigo-visual. De este modo, la selección de un código u otro depende del tipo de operación mental que se requiera en cada caso. En este sentido, la covarianza de error que se presenta entre las tareas de comparación de magnitudes y secuencias numéricas, en la Forma A del IPAM, estaría mediatizada por el procedimiento de trascodificación que se produce al recodificar los números presentados de forma arábiga en una codificación análoga de las cantidades. Este proceso de trascodificación se daría cuando los niños comparan dos números presentados para decidir cuál de ellos presenta mayor magnitud. En CN la presencia de este proceso parece bastante evidente, ya que la tarea se basa en que el alumno seleccione el número mayor de entre dos presentados. En cuanto a $\mathrm{SN}$, dada una secuencia numérica, que se presenta en orden directo o inverso y cuya incógnita varía, decidir qué número de los 
presentados es menor y cuál es mayor sería decisivo para poder determinar qué número completa la misma.

\section{IPAM Forma $B$}

En la Tabla 3 presentamos los análisis estadísticos descriptivos de cada una de las tareas que componen el IPAM (Forma B). Se muestran los valores máximo y mínimo, medias, desviación estándar, asimetría y curtosis para cada tarea. Un primer análisis de estos datos muestra que los resultados presentan una dispersión satisfactoria para todas las tareas salvo para OUD y VP. Los valores de asimetría son cercanos a cero lo que contribuye a una distribución normal de los resultados con la excepción de OUD y VP, los cuales tienden a valores superiores al promedio. Los coeficientes de curtosis se aproximan a cero, a excepción de OUD y VP, las cuales presentan valores por encima de la unidad. A pesar de que los valores obtenidos por OUD y VP en los estadísticos de asimetría y curtosis son superiores a uno, podemos observar que no existen problemas con la distribución de los datos puesto que los valores absolutos de estos índices no exceden los valores 3.0 y 10.0 respectivamente (Kline, 2005). Las desviaciones en las puntuaciones estándar se sitúan entre 10.06 y 3.07.

Tabla 3. Estadísticos descriptivos de IPAM Forma B

\begin{tabular}{|c|c|c|c|c|c|c|}
\hline & Mínimo & Máximo & Media & Desviación estándar & Asimetría & Curtosis \\
\hline $\mathrm{CN}$ & 1 & 61 & 31.04 & 10.96 & -.39 & .19 \\
\hline ODD & 0 & 16 & 4.40 & 3.07 & .68 & .52 \\
\hline SN & 0 & 21 & 10.44 & 4.79 & -.18 & -.53 \\
\hline OUD & 0 & 31 & 9.82 & 4.75 & 1.02 & 2.73 \\
\hline VP & 0 & 18 & 3.65 & 3.52 & 1.19 & 1.28 \\
\hline
\end{tabular}

La matriz de correlación de los resultados obtenidos en las diferentes tareas que componen el IPAM (Forma B) se presenta en la tabla 4. Los resultados obtenidos muestran correlaciones estadísticamente significativas entre todos los indicadores.

Tabla 4. Coeficientes de correlación entre las tareas de IPAM Forma B

\begin{tabular}{lccccc}
\hline & CN & ODD & SN & OUD & VP \\
\hline CN & 1.00 & $.58^{* *}$ & $.48^{* *}$ & $.54^{* *}$ & $.39^{* *}$ \\
\hline ODD & & 1.00 & $.52^{* *}$ & $.67^{* *}$ & $.45^{* *}$ \\
\hline SN & & & 1.00 & $.46^{* *}$ & $.43^{* *}$ \\
\hline OUD & & & & 1.00 & $.41^{* *}$ \\
\hline VP & & & & 1.00 \\
\hline Nota. CN=Comparación de & magnitudes, \\
ODD=Operaciones de dos dígitos, SN=Series \\
numéricas, OUD=Operaciones de un dígito, \\
VP=Valor de posición. IPAM=Indicadores de \\
Progreso de Aprendizaje en Matemáticas. \\
$* * p<.001$.
\end{tabular}


Para la descripción de los resultados obtenidos en el AFC de la Forma B del IPAM se ha realizado la misma interpretación que para la Forma A en cuanto a la representación de las relaciones estructurales observadas en la matriz derivada y a los coeficientes de regresión multivariada. Siendo el factor latente sentido numérico y los indicadores observables las tareas, se encuentra $\mathrm{CN}$ con un coeficiente de 0.66, ODD con un coeficiente de 0.88 , SN con un coeficiente de 0.60 , OUD con un coeficiente de 0.77 , y VP con un coeficiente de 0.51 (ver figura 2).

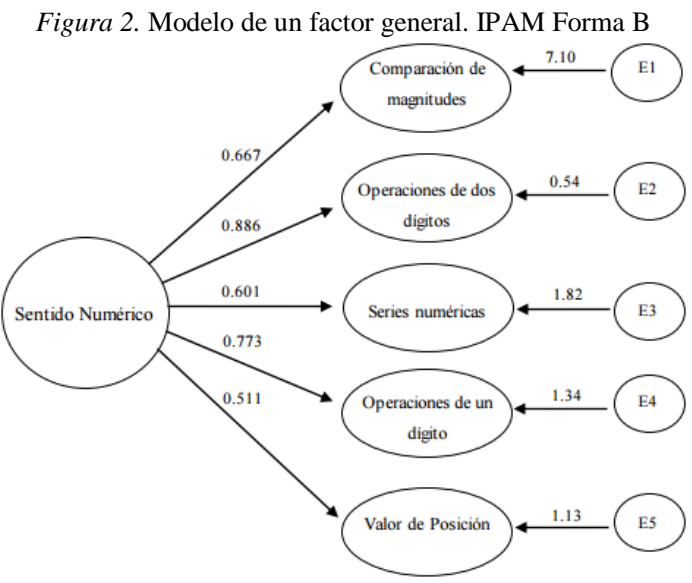

Estos resultados muestran que la evaluación preliminar del modelo verifica que los coeficientes derivados presentan el sentido positivo esperado de acuerdo con la teoría empleada en la configuración del modelo de medida.

Posteriormente, se analizó el nivel de ajuste del modelo propuesto con el modelo estudiado. El valor de $\chi^{2}=2.015$ ( $p=.73$ ), implica que el modelo y los resultados se ajustan entre sí. Los índices de bondad de ajuste confirman el ajuste del modelo $\left(\mathrm{SRMR}=0.017 ; \quad \chi^{2}=2.015 ; \quad d f=4 ; \quad\right.$ pvalue=0.733; $\mathrm{NFI}=0.993 ; \quad \mathrm{CFI}=1 ; \quad \mathrm{GFI}=0.995$; AGFI=0.98; RMSEA=0; IC=0-0.088).

Para que este modelo se ajustase fue necesario liberar la covarianza de error existente entre SN y VP. Esta covarianza, al igual que en la forma A del IPAM, estaría explicada por este proceso de trascodificación. Observamos que cuando el niño se enfrenta a los números que componen la secuencia numérica pasa de un código arábigo a un código análogo de las cantidades. Sin embargo, a continuación, debe volver a realizar una trascodificación del código análogo al arábigo, pues debe completar la serie numérica. Este último procedimiento se da también en la tarea de valor de posición, donde a partir de una representación analógica dada el niño realiza una trascodificación al código arábigo-visual. De este modo, vemos como en ambas tareas se da una recodificación del 
código empleado, pasando de un código análogo de las magnitudes a un código arábigovisual.

\section{IPAM Forma $C$}

En la Tabla 5 presentamos los análisis estadísticos descriptivos de cada una de las tareas que componen el IPAM Forma C. Se muestran los valores máximo y mínimo, medias, desviación estándar, asimetría y curtosis para cada tarea. Un primer análisis de estos datos muestra que los resultados presentan una dispersión satisfactoria para todas las tareas salvo para OUD. Los valores de asimetría y curtosis son cercanos a cero en todas las tareas a excepción de OUD. Los valores obtenidos por OUD para estos estadísticos tienden a valores superiores al promedio, aunque se mantienen dentro de los rangos aceptables (Kline, 2005). Las desviaciones en las puntuaciones estándar se sitúan entre 9.41 y 3.49 .

Tabla 5. Estadísticos descriptivos de IPAM Forma C

\begin{tabular}{lcccccc}
\hline & Mínimo & Máximo & Media & Desviación estándar & Asimetría & Curtosis \\
\hline CN & 2 & 57 & 36.12 & 9.41 & -.83 & 1.81 \\
\hline ODD & 0 & 16 & 7.42 & 3.49 & .42 & -.22 \\
\hline SN & 0 & 30 & 12.82 & 5.53 & .03 & .18 \\
\hline OUD & 1 & 32 & 11.58 & 5.16 & 1.06 & 2.13 \\
\hline VP & 0 & 22 & 7.20 & 4.87 & .46 & -.65 \\
\hline Nota. CN=Comparación de magnitudes, ODD=Operaciones de dos dígitos, SN=Series \\
numéricas, OUD=Operaciones de un dígito, VP=Valor de posición. IPAM=Indicadores de \\
Progreso de Aprendizaje en Matemáticas.
\end{tabular}

La matriz de correlación de los resultados obtenidos en las diferentes tareas que componen el IPAM Forma $\mathrm{C}$ se presenta en la tabla 6. Los resultados obtenidos muestran correlaciones estadísticamente significativas entre todos los indicadores.

Tabla 6. Coeficientes de correlación entre las tareas de IPAM Forma C

\begin{tabular}{llllll}
\hline & CN & ODD & SN & OUD & VP \\
\hline CN & 1.00 & $.39^{* *}$ & $.43^{* *}$ & $.44^{* *}$ & $.39^{* *}$ \\
\hline ODD & & 1.00 & $.49^{* *}$ & $.59^{* *}$ & $.49^{* *}$ \\
\hline SN & & 1.00 & $.54^{* *}$ & $.52^{* *}$ \\
\hline OUD & & & 1.00 & $.55^{* *}$ \\
\hline VP & & & & 1.00 \\
\hline Nota. CN=Comparación de & magnitudes, \\
ODD=Operaciones de dos dígitos, SN=Series \\
numéricas, OUD=Operaciones de un dígito, \\
VP=Valor de posición. IPAM=Indicadores de \\
Progreso de Aprendizaje en Matemáticas. \\
$* * p<.001$.
\end{tabular}

Para la descripción de los resultados obtenidos en el AFC de la Forma C del IPAM se ha realizado la misma interpretación que para la Forma A en cuanto a la representación de las relaciones estructurales observadas en la matriz derivada y a los coeficientes de regresión multivariada. Siendo el factor latente sentido numérico y los 
indicadores observables las tareas, se encuentra $\mathrm{CN}$ con un coeficiente de 0.54, ODD con un coeficiente de 0.70 , SN con un coeficiente de 0.70 , OUD con un coeficiente de 0.78 , y VP con un coeficiente de 0.70 (ver figura 3 ).

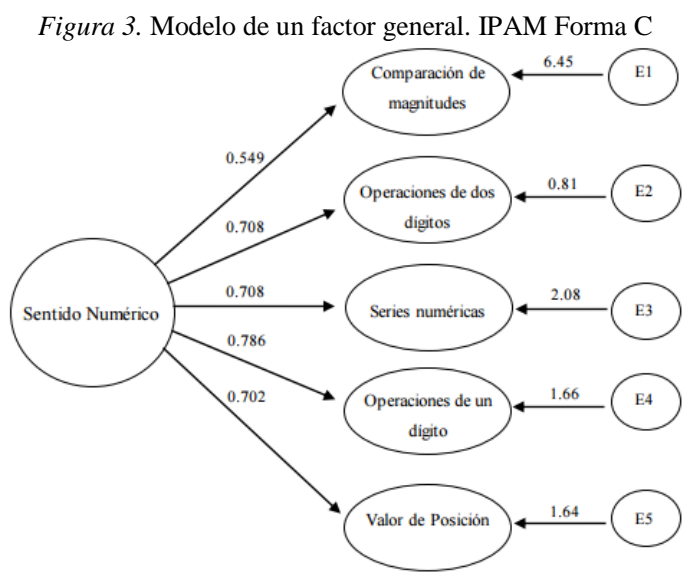

Estos resultados muestran que la evaluación preliminar del modelo verifica que los coeficientes derivados presentan el sentido positivo esperado de acuerdo con la teoría empleada en la configuración del modelo de medida.

Posteriormente, se analizó el nivel de ajuste del modelo propuesto con el modelo estudiado. El valor de $\chi^{2}=2.555(p=.76)$, implica que el modelo y los resultados se ajustan entre sí. Los índices obtenidos a través del análisis de la bondad de ajuste confirman el ajuste del modelo $\left(\mathrm{SRMR}=0.017 ; \chi^{2}=2.555 ; d f=5\right.$; pvalue $=0.768 ; \mathrm{NFI}=0.99$; $\mathrm{CFI}=1 ; \mathrm{GFI}=0.994 ; \mathrm{AGFI}=0.981 ; \mathrm{RMSEA}=0 ; \mathrm{IC}=0-0.073)$.

\section{DISCUSIÓN Y CONCLUSIONES}

En este artículo se ha pretendido poner a prueba un modelo hipotético para profundizar en la dimensionalidad del sentido numérico mediante la técnica de Análisis Factorial Confirmatorio (AFC). Los datos obtenidos y el análisis de la estructura factorial siguiendo la técnica AFC permiten verificar un buen ajuste del modelo propuesto donde, siguiendo la técnica AFC, se ha confirmado que la estructura de los datos se ha podido obtener también empíricamente. Por tanto, el factor latente Sentido Numérico explica cada uno de los indicadores observables (i.e. comparación de magnitudes, operaciones de un dígito, operaciones de dos dígitos, series numéricas, y valor de posición), para cada uno de los momentos de medida (i.e., noviembre, febrero, mayo). 
Estos resultados se ajustan a lo prescrito por la teoría en cuanto a la definición de este constructo y las habilidades que lo conforman, incluyendo las habilidades tempranas típicamente asociadas con el desarrollo del sentido numérico (Griffin, 2004; Jordan, Kaplan, Oláh, y Locuniak, 2006; NMAP, 2008).

Son diversos los autores que han diseñado herramientas para evaluar el rendimiento en matemáticas del alumnado en Educación Primaria basándose en el sentido numérico. Lembke y Foegen (2009) desarrollaron una herramienta CBM, para la cual utilizaron como indicadores las tareas discriminación de cantidades, discriminación visual de magnitudes, e identificación numérica. Estas dos últimas tareas resultaron ser mejores predictores del estatus de riesgo. En un estudio realizado por Clarke y Shinn (2004), se encontró que el mejor predictor del estatus de riesgo era la tarea discriminación de cantidades. Los mismos resultados fueron encontrados por Clarke, Baker, Smolkowski, y Chard (2008). En una revisión realizada por Foegen et al. (2007) concluyeron que las tareas que mayor soporte científico presentan para el diseño de herramientas de evaluación temprana de las matemáticas son identificación numérica, discriminación de cantidades y averiguar el número que falta en una secuencia. Del mismo modo, señalan que las medidas más adecuadas para la evaluación del rendimiento en matemáticas en educación primaria son cálculo y conceptos y aplicaciones (i.e. lectura de gráficos y tablas, comprensión del número y resolución de problemas).

Nuestra principal aportación con la presente investigación ha sido la de contrastar un modelo basado en la teoría donde la parte que cada una de las tareas seleccionadas comparte entre sí puede atribuirse al sentido numérico como estructura latente no observada. No obstante, una limitación del presente estudio hace referencia al conjunto de tareas que han sido seleccionadas ya que no podemos asegurar que otras tareas puedan también ser igualmente válidas para la medición del sentido numérico.

Como conclusión establecemos que el IPAM presenta una adecuada validez de constructo ya que permite apresar la capacidad de sentido numérico en alumnado del primer curso de educación primaria. A la vista de la revisión de estudios, se ha puesto de manifiesto que esta capacidad estaría a la base de las dificultades que pueden experimentar algunos niños que están aprendiendo las matemáticas. Por tanto, líneas futuras de investigación han de centrarse, por un lado, en constatar la validez de constructo en los siguientes cursos de primaria (i.e., segundo y tercer curso) y, por otro lado, en analizar la sensibilidad y especificidad del IPAM para la detección de alumnado en riesgo de presentar DAM, así como su validez a la hora de evaluar el progreso de aprendizaje.

\section{Agradecimientos}

Este trabajo ha sido posible gracias a la financiación del Plan Nacional I+D+i del Ministerio de Economía y Competitividad, con ref. EDU2012-35098, siendo el IP el primer autor. 


\section{REFERENCIAS}

Andrews, P., y Sayers, J. (2015). Identifying opportunities for grade one children to acquire foundational number sense: Developing a framework for cross-cultural classroom analyses. Early Childhood Education Journal, 43, 257-267.

Aubrey, C., Dahl, S., y Godfrey, R. (2006). Early mathematics development and later achievement: Further evidence. Mathematics Education Research Journal, 18(1), 27-46.

Aunio, P., y Niemivirta, M. (2010). Predicting children's mathematical performance in grade one by early numeracy. Learning and Individual Differences, 20(5), 427-435.

Baker, S., Gersten, R., y Lee, D. (2002). A synthesis of empirical research on teaching mathematics to low-achieving students. The Elementary School Journal, 103(1), 51-73.

Barth, H., Beckmann, L., y Spelke, E.S. (2008). Nonsymbolic, approximate arithmetic in children: Abstract addition prior instruction. Developmental Psychology, 44(5), 1466-1477.

Bruer, J.T. (1997). Education and the brain: A bridge too far. Educational Researcher, 26 (8), 4-16.

Clarke, B., Baker, S., Smolkowski, K., y Chard, D.J. (2008). An analysis of early numeracy curriculum-based measurement. Examining the role of growth in student outcomes. Remedial and Special Education, 29(1), 49-57.

Clarke, B., y Shinn, M.R. (2004). A preliminary investigation into the identification and development of early mathematics curriculum-based measurement. School Psychology Review, 33(2), 234-248.

Conderman, G., Jung, M., y Hartman, P. (2014). Subitizing and early mathematics standards: A winning combination. Kappa Delta Pi Record, 50(1), 18-23.

Dehaene, S., y Cohen, L. (1995). Towards an anatomical and functional model of number processing. Mathematical Cognition, 1, 83-120.

Dehaene, S., Piazza, M., Pinel, P., y Cohen, L. (2003). Three parietal circuits for number processing. Cognitive Neuropsychoy, 20, 487-506.

Foegen, A., Jiban, C., y Deno, S. (2007). Progress monitoring measures in mathematics. A review of the literature. The Journal of Special Education, 4(2), 121-139.

Fornell, C., y Larcker, D.F. (1981). Evaluating structural equation models with unobservable variables and measurement error. Journal of Marketing Research 18(1), 39-50.

Fuchs, L.S., y Fuchs, D. (1986). Effects of systematic formative evaluation: A meta-analysis. Exceptional Children, 53(3), 199-208.

Geary, D. (2013). Early foundations for mathematics learning and their relations to learning disabilities. Current Directions in Psychological Science, 22(1), 23-27.

Gersten, R., y Chard, D. (1999). Number Sense: Rethinking arithmetic instruction for students with mathematical disabilities. The Journal of Special Education, 33(1), 18-28.

Gersten, R., Jordan, N.C., y Flojo, J.R. (2005). Identification and intervention for students with mathematics difficulties. Journal of Learning Disabilities, 38(4), 293-304.

Griffin, S. (2004). Building number sense with number worlds: a mathematics program for young children. Early Childhood Research Quarterly, 19, 173-180.

Ivrendi, A. (2011). Influence of self-regulation on the development of children's number sense. Early Childhood Education Journal, 39(4), 239-247.

Jiménez, J.E., y de León, S.C. (2016). Indicadores de progreso de aprendizaje en matemáticas (IPAM). Universidad de La Laguna.

Jordan, N.C., Kaplan, D., Locuniak, M.N., y Ramineni, C. (2007). Predicting first-grade math achievement from developmental number sense trajectories. Learning Disabilities Research \& Practice, 22(1), 36-49. 
Jordan, N.C., Kaplan, D., Oláh, L.N., y Locuniak, M.N. (2006). Number sense growth in kindergarten: A longitudinal investigation of children at risk for mathematics difficulties. Child Development, 77(1), 153-175.

Jordan, N., y Levine, S. (2009). Socioeconomic variation, number competence, and mathematics learning difficulties in young children. Developmental Disabilities Research Reviews, 15(1), 60-68.

Jöreskog, K., y Sorbom, D. (1996-2001). LISREL 8: User's Reference Guide. Illinois: Scientific Software International, Lincolnwood.

Jung, M., Hartman, P., Smith, T., y Wallace, S. (2013). The effectiveness of teaching number relationships in preschool. International Journal of Instruction, 6 (1), 165-178.

Kelley, B., Hosp, J.L., y Howell, K.W. (2008). Curriculum-based evaluation and math. An overview. Assessment for Effective Intervention, 33(4), 250-256.

Kline, R.B. (2005). Principles and practice of structural equation modeling (2nd ed.). Nueva York, NY: Guilford Press.

Kolkman, M.E., Kroesbergen, E.H., y Leseman, P.P.M. (2013). Early numerical development and the role of nonsymbolic and symbolic skills. Learning and Instruction, 25, 95-103.

LeFevre, J.A., Fast, L., Skwarchuk, S.L., Smith-Chant, B.L., Bisanz, J., Kamawar, D., y Penner-Wilger, M. (2010). Pathways to mathematics: Longitudinal predictors of performance. Child Development, 81, 1753-1767.

Lembke, E., y Foegen, A. (2009). Identifying early numeracy indicators for kindergarten and firstgrade students. Learning Disabilities Research \& Practice, 24, 12-20.

Mazzocco, M.M., y Thompson, R.E. (2005). Kindergarten predictors of math learning disability. Learning Disabilities Research \& Practice, 20(3), 142-155.

Nieder, A., y Dehaene, S. (2009). Representation of number in the brain. Annual Review in Neuroscience, 32, 185-208.

National Council of Teachers of Mathematics. (2000). Principles and standards for school mathematics. Reston, VA: NCATE.

National Mathematics Advisory Panel (2008). Foundations for success: The final report of the National Mathematics Advisory Panel. Washington, DC: Department of Education.

Sasanguie, D., Göbel, S.M., Moll, K., Smets, K., y Reynvoet, B. (2013). Approximate number sense, symbolic number processing, or number-space mappings: What underlies mathematics achievement? Journal of Experimental Child Psychology, 114, 418-431.

Sood. S., y Jitendra, A.K. (2007). A comparative analysis of number sense instruction in reformbased and traditional mathematics textbooks. The Journal of Special Education, 41(3), 145-157.

Stecker, P.M., Fuchs, L.S., y Fuchs, D. (2005). Using curriculum-based measurement to improve student achievement: Review of research. Psychology in the Schools, 42(8), 795- 819.

$\mathrm{Xu}, \mathrm{F}$., y Arriaga, R.I. (2007). Number discrimination in 10-month-old infants. British Journal of Developmental Psychology, 25, 103-108.

Recibido: 23 de septiembre de 2016

Recepción Modificaciones: 1 de febrero de 2017 Aceptado: 4 de febrero de 2017 